\title{
ATMOSPHERIC CORRECTIONS OF LOW ALTITUDE THERMAL INFRARED AIRBORNE IMAGES ACQUIRED OVER A TROPICAL CROPPED AREA
}

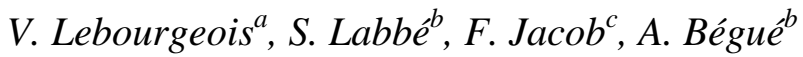 \\ ${ }^{a}$ CIRAD, UR SCA, Chemin de Grand Canal, BP20, 97408 Saint Denis Messagerie Cedex, Ile de La \\ Réunion, France - valentine.lebourgeois@cirad.fr \\ ${ }^{\mathrm{b}}$ UMR TETIS (CEMAGREF-CIRAD-ENGREF), 500 rue J-F. Breton, 34093 Montpellier Cedex 5, \\ France, - (sylvain.labbe, begue)@teledetection.fr \\ ${ }^{c}$ UMR LISAH (SupAgro-INRA-IRD), 2 place Pierre Viala, 34060 Montpellier Cedex 1, France, - \\ frederic.jacob@supagro.inra.fr
}

\begin{abstract}
Accurate corrections of atmospheric effects on thermal infrared remote sensing data are an essential pre-requisite for the development of thermal infrared airborne-derived crop water stress indices. These corrections can be performed using ground surface temperature measurements, which are time consuming and expensive. Atmospheric effects can also be corrected using radiative transfer models that require knowledge of atmospheric status. The latter can be accurately characterized from radiosoundings, but these are usually unavailable. It can also be derived from meteorological model simulations, but spatial and temporal resolution are often too coarse. This study proposes performing atmospheric corrections by using temperature and relative humidity profiles acquired in flight from onboard sensors during data collection. Such measurements are used to document the atmospheric radiative transfer model MATISSE. First results from an experimentation over a tropical cropped area show that corrections are made with a $1.46^{\circ} \mathrm{K}$ accuracy.
\end{abstract}

Index Terms - Atmospheric corrections, Thermal Infrared, Airborne images, Matisse software.

\section{INTRODUCTION}

Surface temperature of canopy is known to be strongly related with the water status of the crop and is widely used for development of water deficit indices ([3], [6]). Several factors affect the thermal infrared response of a vegetative canopy as its temperature is both determined by external environmental factors and by internal plant factors ([2]). The availability of bolometric cameras at affordable price used in combination with ultralight aircraft or unmanned aerial vehicle pave the way for new water stress survey methods in precision farming applications.
When remotely sensed from airborne or spaceborne sensors, the thermal infrared signal emitted by crops has to be corrected for atmospheric effects ([5]). These corrections can be derived from ground surface temperature measurements, which is tedious and expensive. They can also be calculated from radiative transfer simulations. The latter however require accurate information about atmospheric status between the target and the sensor, such as vertical profiles of temperature, humidity, pressure, and atmospheric content in aerosols and molecular gazes ([4]). Atmospheric profiles can be derived from radiosoundings, but these are usually unavailable or do not coincide in time or in space. They can also be derived from atmospheric models after reanalysis procedures, but the spatial and temporal samplings (100 km and 6 hours) are too coarse. Moreover, changes of low-altitude atmospheric conditions can occur rapidly in tropical zones and near real-time parameters should be taken into account for adequately correct the atmospheric effects.

This paper presents a method to correct the atmospheric perturbations on thermal measurements collected from on board cost effective sensors, by using measurements of air temperature and relative humidity at different altitudes during the flight. Radiative transfer function is computed using the MATISSE (Advanced Earth Modeling for Imaging and Scene Simulation, [1]) software. The experiment was conducted in Reunion Island (a French tropical overseas department located in the Indian Ocean), over sugarcane fields in 2007. First results indicate that without requiring ground-truth surface temperature measurements or radiosounding data, this method could provide an alternative cost-efficient correction of atmospheric effects with enough accuracy for crop stress monitoring. 


\section{DATA ACQUISITION}

Airborne and ground acquisitions were performed during 5 months, twice a month, over an experimental sugarcane field. In order to capture large temperature contrasts, flights occurred around solar noon during sunny days.

\subsection{The airborne acquisition system}

The airborne acquisition system consisted of an Ultra Light Aircraft equipped with a B20 HSV FLIR micro-bolometer thermal infrared camera (Fig.1.a). The radiance, detected over the [7.5 - 15] $\mu \mathrm{m}$ spectral interval, is expressed in equivalent temperature assuming a target emissivity equal to unity. The system provided 240 pixel x 320 pixel images with a radiometric resolution of $0.1^{\circ} \mathrm{K}$ and an absolute precision of $2^{\circ} \mathrm{K}$. To achieve the meteorological and location measurements, a temperature and relative humidity probe (Fig.1.b) coupled to a data logger was loaded in the Ultra-Light Aircraft, as well as a GPS that recorded the altitude and position during the flight. These measurements are next used to generate temperature and relative humidity profiles as functions of altitude. Images were taken from 300 $\mathrm{m}$ to $1300 \mathrm{~m}$ in order to study the sensitivity of the signal to atmospheric transmittance.
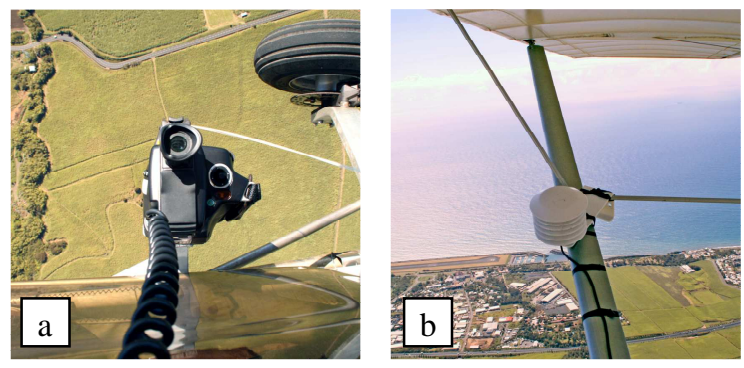

Fig.1: (a) Thermal infrared camera B20HSV, Flir Systems ${ }^{\circledR}$, (b) Temperature and relative humidity sensor, HMP50 Campbell $^{\circledR}$

\subsection{Ground acquisitions}

Ground data were simultaneously acquired with airborne acquisitions on cold (sugarcane canopy), hot (dark tissue or stone road) and intermediate (grassy road) targets (see example in Fig.2). The instrument used was a hand-held infrared thermometer HEITRONICS ${ }^{\circledR}$ KT19 that measured the target temperature from the incoming radiance over the [8 - 14] $\mu \mathrm{m}$ spectral interval, by assuming a target emissivity of 1 . The lens FOV was about $13.5^{\circ}$. Due to the sugarcane height, the thermometer was mounted on a mast for vertical acquisitions over the canopy, such as angular variation between airborne and in situ measurements were avoided (Fig.3). Consequently, the measurements provided a circular footprint diameter of approximately $25 \mathrm{~cm}$ on sugarcane (cold) target, and $95 \mathrm{~cm}$ on hot and intermediate temperature targets. Air temperature and humidity were also recorded with the same temperature and relative humidity probe as on the aircraft.

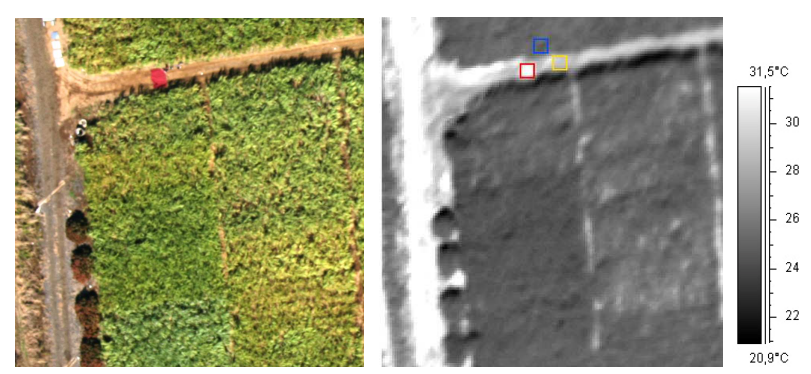

Fig.2: Location of the targets on a visible (left) and thermal infrared image (right). Targets are surrounded in red (hot), yellow (intermediate) and blue (cold) color.

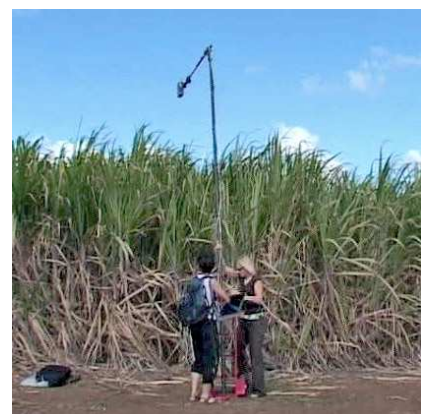

Fig.3: In-situ measurements of surface temperature using a hand-held infrared thermometer HEITRONICS KT19 ${ }^{\circledR}$ mounted on a mast.

\section{DATA ANALYSIS AND RESULTS}

\subsection{Sensors calibration}

Both thermal infrared sensors (KT19 and B20HSV camera) were calibrated against a blackbody for temperatures ranging between 290 and $315{ }^{\circ} \mathrm{K}$. The calibration results obtained from the thermal infrared camera B20HSV were consistent with those obtained from the KT19 sensor. Mean of different measurements was $0.15^{\circ} \mathrm{K}$ between the two sensors and both sensors were always better than $0.4^{\circ} \mathrm{K}$ accurate in reference to the blackbody absolute temperature. Therefore, no corrections were applied to the temperature values given by the sensors.

\subsection{Data Analysis}

First of all we tried to linearly relate the ground thermal infrared measurements and the airborne thermal images for each acquisition date. Thus the mean temperature of each target was calculated from airborne data set and compared against ground-truth measurements. As displayed by Fig.4 
for a given acquisition date, the linear regressions showed that measured temperature of surface objects decreased with altitude, which was explained by the attenuation of surface signal when surface is hotter than atmosphere. The constant term of the regressions increased with altitude, which was explained by the atmospheric signal proportion of the measurements. For measured temperatures closer to air temperature, altitude effects were lower as can be seen in Fig.4 for cold targets (surrounded with blue color).

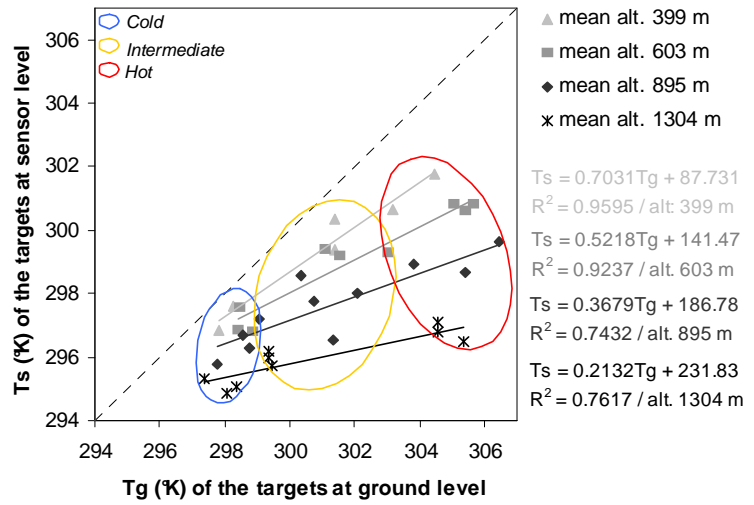

Fig.4: Relation between surface ( $\mathrm{Tg}$ ) and sensor airborne temperature (Ts) measurements acquired on $27^{\text {th }}$ July 2007 at different altitudes. Colored circles indicate the type of target. Linear regressions for each altitude are plotted with a color gradient from light grey (lowest altitude) to black (highest altitude).

Those results (i.e. differences between surface and sensor level temperatures up to $9{ }^{\circ} \mathrm{K}$ ) showed, as expected, the necessity to correct the airborne thermal infrared images from the atmospheric effects in order to accurately retrieve the actual surface temperature.

\subsection{Variability of atmospheric effects}

The linear regressions previously presented could be used to perform a coarse correction for a specific acquisition date. However, the terms of the linear regression between $\mathrm{Tg}$ and Ts, for a given altitude, were different from a date to another (Fig.5), with variations due to changes in atmospheric conditions (pressure, temperature and humidity). Thus, a global atmospheric correction model, based on linear regressions could not be used.

As the acquisition of ground surface temperatures on cold and hot targets simultaneously with the flights is not feasible for an operational monitoring of large cropped areas, another way to correct atmospheric effects is recommended. When using radiative transfer models, several difficulties related to estimation of atmospheric parameters are encountered. The use of radiosoundings is not easy because of unavailability or non coincidence in time or space. The use of atmospheric models after reanalysis procedures is also questionable because of coarse spatial and temporal resolution (currently $100 \mathrm{~km}$ and 6 hours). Therefore, another way to carry out atmospheric corrections is proposed here, based on atmospheric radiative transfer simulations to be documented with in flight measurements of air temperature and humidity. This is presented in the following part.

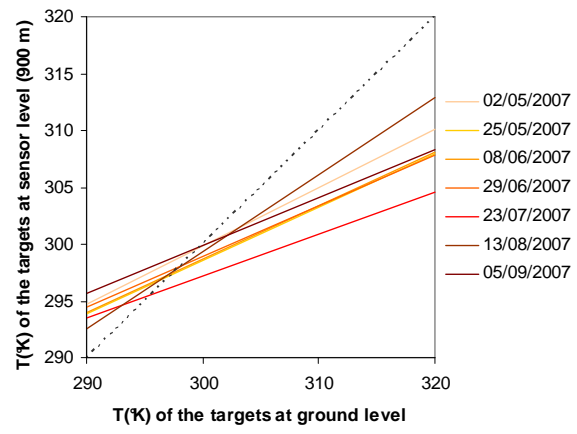

Fig.5: Relation between surface ( $\mathrm{Tg}$ ) and airborne sensor temperature for a given altitude $(900 \mathrm{~m})$ at different acquisition dates.

\subsection{Matisse simulations}

Atmospheric effects were studied using the Matisse 1.5.0 atmospheric radiative transfer model along with pressure, air temperature and relative humidity profiles. These profiles generally come from radiosoundings, that were, in our case, unavailable for the whole duration of the experimentation, except for one date (05/09/2007). Thus, for each acquisition date, temperature and relative humidity profiles were acquired with an on-board probe (HMP50), during the flight durations (i.e. approximately one hour).

For 05/09/2007 acquisition date, Fig. 6 shows that the two profiles acquired by the on-board probes and by the radiosounding (launched 1 hour and 45 minutes before airborne data acquisition $2 \mathrm{~km}$ away) were in good agreement, illustrating a satisfactory operating of the HMP50 sensor in the flight conditions.

For this date, we used MATISSE to simulate the collected ground temperatures at the sensor level using the two humidity and temperature data sources: the radiosounding and the profile acquired by the on-board probe. Simulated ground based temperatures at the sensor level were then compared against actual airborne measured temperatures. Results (Table.1) show an RMSE of $0.63{ }^{\circ} \mathrm{K}$ for the first one and $0.49{ }^{\circ} \mathrm{K}$ for the second one, highlighting a better accuracy for the solution implemented to replace radiosounding data.

The same comparison was performed between results from Matisse simulations of ground based temperatures along with airborne atmospheric profiles and actual thermal camera measurements for all acquisition dates and altitudes (Fig. 7). Simulated temperatures were in agreement with 
those acquired with the on-board camera. The root mean square errors, calculated for each type of targets (Table.2), showed an accuracy of $1.05{ }^{\circ} \mathrm{K}$ for cold target, which corresponds to a sugarcane canopy. A larger drift were however noticed as target temperature increased. On going investigations are focused on understanding this drift for larger temperatures.

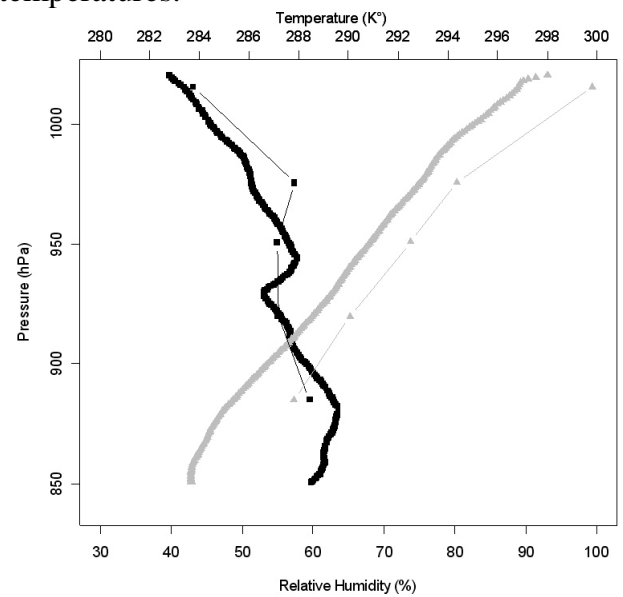

Fig.6: An example (thin lines) of air temperature (grey) and relative humidity (black) profile, acquired during the 5 September 2007 flight at 11:45 a.m. Thick lines correspond to a radiosounding profile launched the same day at 10:00 a.m. at Gillot airport.

\begin{tabular}{l|c|c|}
\multicolumn{1}{c}{} & \multicolumn{2}{c}{ MATISSE simulations with: } \\
& Airborne profile & Radiosounding \\
\hline RMSE Total (K) & $\mathbf{0 . 4 9}$ & $\mathbf{0 . 6 3}$ \\
RMSE Cold target (K) & 0.63 & 0.82 \\
RMSE Intermediate target (K) & 0.30 & 0.35
\end{tabular}

Table.1: Root Mean Square Errors between actual airborne measured temperatures and temperature simulated with two different atmospheric profiles.

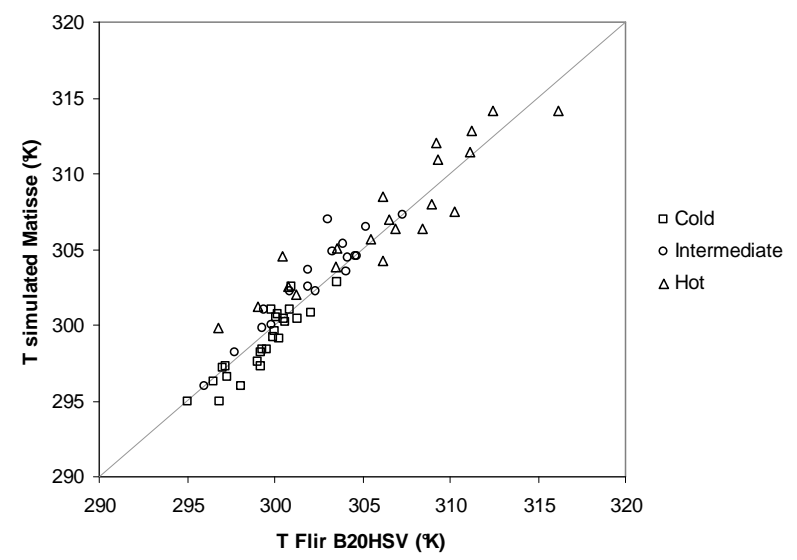

Fig.7: Relation between the temperature at sensor level ( $\mathrm{T}$ Flir B20HSV) and the temperature simulated with Matisse software for the whole data set.

\begin{tabular}{l|l} 
RMSE Total (K) & $\mathbf{1 . 4 6}$ \\
\hline RMSE Cold target (K) & 1.05 \\
RMSE Intermediate target (K) & 1.28 \\
RMSE Hot target (K) & 1.94
\end{tabular}

Table.2: Root Mean Square errors between simulated and actual airborne measured temperatures

\section{CONCLUSION AND OUTLOOKS}

The proposed original method to correct atmospheric effects on thermal airborne images via the use of an atmospheric radiative transfer model along with airborne-derived temperature and humidity profile show a sufficient accuracy for study of crop stress. Future investigations will focus on the potential of such on-board atmospheric information, with various degrees of complexity. This will hopefully provide solutions based on real time characterization of atmospheric status for the corrections of airborne thermal infrared remote sensing data.

\section{AKNOWLEDGMENTS}

ONERA and especially Luc Labarre for help on Matisse Software. INRA - Avignon for sensor calibration. Marion Houlès from CIRAD for ground acquisitions in Reunion Island.

\section{REFERENCES}

[1] P. Simoneau, R. Berton, K. Caillault, G. Durand, T. Huet, L. Labarre, C. Malherbe, C. Miesch, A. Roblin, B. Rosier, "MATISSE, Advanced Earth Modeling for Imaging and Scene Simulation", ESO/SPIE Europto European Symposium on Remote Sensing, Toulouse (France), September 17-21, 10p., 2001.

[2] P. Boissard, G. Guyot, and al., "Factors affecting the radiative temperature of a vegetative canopy," Applications of remote sensing in agriculture, pp. 45-72, 1990.

[3] R. D. Jackson, S. B. Idso, and al., "Canopy temperature as a crop water stress indicator," Water Resource Research, vol. 17, pp. 1133-1138, 1981.

[4] F. Jacob, F. Petitcolin, T. Schmugge, and al., "Comparison of land surface emissivity and radiometric temperature derived from MODIS and ASTER sensors," Remote Sensing of Environment, vol. 90/2, pp. 137-152, 2004.

[5] J. C. Jimenez-Munoz and J. A. Sobrino, "Error sources on the land surface temperature retrieved from thermal infrared single channel remote sensing data," International Journal of Remote Sensing, vol. 27/5-6, pp. 999-1014, 2006.

[6] M. S. Moran, T. R. Clarke, and al., "Estimating crop water deficit using the relation between surface-air temperature and spectral vegetation index," Remote Sensing of Environment, vol. 46/2, pp. 246-263, 1994. 